\title{
Symmetric positive solutions to a second-order boundary value problem with integral boundary conditions
}

\author{
Huihui Pang ${ }^{1 *}$ and Yulong Tong ${ }^{2}$
}

${ }^{*}$ Correspondence:

phh2000@163.com

${ }^{1}$ College of Science, China

Agricultural University, Beijing,

100083, P.R. China

Full list of author information is

available at the end of the article

\begin{abstract}
This paper investigates the existence of concave symmetric positive solutions and establishes corresponding iterative schemes for a second-order boundary value problem with integral boundary conditions. The main tool is a monotone iterative technique. Meanwhile, an example is worked out to demonstrate the main results.
\end{abstract}

Keywords: integral boundary conditions; iterative; monotone positive solution; symmetric; completely continuous

\section{Introduction}

The theory of boundary value problems with integral boundary conditions for ordinary differential equations arises in different areas of applied mathematics and physics. For example, heat conduction, chemical engineering, underground water flow, thermo-elasticity, and plasma physics can be reduced to the nonlinear problems with integral boundary conditions; we refer readers to [1-3] for examples and references.

At the same time, boundary value problems with integral boundary conditions constitute a very interesting and important class of problems. They include two, three, multipoint and nonlocal boundary value problems as special cases.

Hence, increasing attention is paid to boundary value problems with integral boundary conditions [4-8]. Generally, the Guo-Krasnosel' skii fixed point theorem in a cone, the Leggett-Williams fixed point theorem, the method of upper and lower solutions and the monotone iterative technique play extremely important roles in proving the existence of solutions to boundary value problems. In particular, we would like to mention some excellent results.

In [4], Ma studied the following problem:

$$
\left\{\begin{array}{l}
u^{(4)}(t)=h(t) f(t, u), \quad 0<t<1 \\
u(0)=u(1)=\int_{0}^{1} p(s) u(s) d s \\
u^{\prime \prime}(0)=u^{\prime \prime}(1)=\int_{0}^{1} q(s) u(s) d s
\end{array}\right.
$$

where $p, q \in L^{1}[0,1], h$ and $f$ are continuous. The existence of at least one symmetric positive solution is obtained by the application of the fixed point index in cones.

(C) 2013 Pang and Tong; licensee Springer. This is an Open Access article distributed under the terms of the Creative Commons Attribution License (http://creativecommons.org/licenses/by/2.0), which permits unrestricted use, distribution, and reproduction in any medium, provided the original work is properly cited. 
In 2010, Wang et al. [7] considered the second-order boundary value problem with the integral boundary conditions

$$
\left\{\begin{array}{l}
\left(\phi\left(u^{\prime}(t)\right)\right)^{\prime}+f\left(t, u(t), u^{\prime}(t)\right)=0, \quad 0<t<1, \\
u(0)-k_{1} u^{\prime}(0)=\int_{0}^{1} h_{1}(u(s)) d s, \\
u(1)+k_{2} u^{\prime}(1)=\int_{0}^{1} h_{2}(u(s)) d s,
\end{array}\right.
$$

where $\phi, f, h_{1}$ and $h_{2}$ are continuous, $k_{1}$ and $k_{2}$ are nonnegative constants. The existence result was obtained by applying the method of upper and lower solutions and LeraySchauder degree theory. Theorem 1 (see [7]) supposed that the upper and lower solutions exist, and then, the theory of differential inequalities was used to prove that there is a solution to the boundary value problem between the upper and lower solutions.

Different from [7], [9] is not based on the assumption that the upper and lower solutions to the boundary value problem should exist, but constructs the specific form of the symmetric upper and lower solutions. The author in [9] investigated a second-order SturmLiouville boundary value problem

$$
\begin{cases}w^{\prime \prime}(t)+h(t) f(w(t))=0, & 0<t<1, \\ \alpha w(0)-\beta w^{\prime}(0)=0, & \alpha w(1)+\beta w^{\prime}(1)=0 .\end{cases}
$$

And by applying monotone iterative techniques, author proved the existence of $\mathrm{n}$ symmetric positive solutions.

To the best of our knowledge, no contribution exists concerning the existence of solutions for a boundary value problem with integral boundary conditions by applying monotone iterative techniques. Inspired by the work mentioned above, we concentrate on the following problem:

$$
\begin{aligned}
& u^{\prime \prime}(x)+f\left(x, u(x), u^{\prime}(x)\right)=0, \quad 0<x<1, \\
& u(0)=u(1)=\int_{0}^{1} p(s) u(s) d s,
\end{aligned}
$$

where $p \in L^{1}[0,1]$. We construct a specific form of the symmetric upper and lower solutions, and by applying monotone iterative techniques, we construct successive iterative schemes for approximating solutions.

The difficulty of this paper is that the nonlinear term $f$ depends on $u^{\prime}$, which leads to complexities to prove the properties of the operator $T$, especially the monotonicity of the operator $T$. In Lemma 2.2, we skillfully use the cone's character to overcome the mentioned obstacle. In addition, it is worth stating that the first term of our iterative scheme is a simple function or a constant function. Therefore, the iterative scheme is feasible. Under the appropriate assumptions on nonlinear term, this paper aims to establish a new and general result on the existence of a symmetric positive solution to BVP (1.1) and (1.2).

\section{Preliminaries}

Definition 2.1 Let $E$ be a Banach space, a nonempty convex closed set $P \subset E$ is said to be a cone provided the following hypotheses are satisfied: 
(i) if $u \in P, \lambda \geq 0$, then $\lambda u \in P$;

(ii) if $u \in P$ and $-u \in P$, then $u=0$.

Every cone $P \subset E$ induces a partial ordering ' $\leq$ ' on $E$ defined by

$$
u \leq v \quad \text { if and only if } \quad v-u \in P .
$$

Definition 2.2 Let $(E, \leq)$ be an ordered Banach space. An operator $\varphi: E \rightarrow E$ is said to be nondecreasing (nonincreasing) provided that $\varphi(u) \leq \varphi(v)(\varphi(u) \geq \varphi(v))$ for all $u, v \in E$ with $u \leq v$. If the inequality is strict, then $\varphi$ is said to be strictly nondecreasing (nonincreasing).

Definition 2.3 Let $E=C^{1}[0,1], u \in E$ is said to be concave on $[0,1]$ if

$$
u\left(\lambda x_{1}+(1-\lambda) x_{2}\right) \geq \lambda u\left(x_{1}\right)+(1-\lambda) u\left(x_{2}\right)
$$

for any $x_{1}, x_{2} \in[0,1]$ and $\lambda \in[0,1]$.

We consider the Banach space $E=C^{1}[0,1]$ equipped with the norm $\|u\|=\max \left\{\|u\|_{\infty}\right.$, $\left.\left\|u^{\prime}\right\|_{\infty}\right\}$, where $\|u\|_{\infty}=\max _{x \in[0,1]}|u(x)|$. In this paper, a symmetric positive solution $u^{*}$ of (1.1) means a function which is symmetric and positive on $(0,1)$ and satisfies equation (1.1) as well as the boundary conditions (1.2).

In this paper, we always suppose that the following assumptions hold:

$\left(\mathrm{H}_{1}\right) f \in C([0,1] \times[0,+\infty) \times R,[0,+\infty)), f(x, u, v)=f(1-x, u,-v)$ for $x \in\left[\frac{1}{2}, 1\right]$, and $f(x, u, v)>0$ for all $(x, u, v) \in[0,1] \times[0,+\infty) \times R$;

$\left(\mathrm{H}_{2}\right) f(x, \cdot, v)$ is nondecreasing for each $(x, v) \in\left[0, \frac{1}{2}\right] \times R, f(x, u, \cdot)$ is nondecreasing for $(x, u) \in\left[0, \frac{1}{2}\right] \times[0,+\infty) ;$

$\left(\mathrm{H}_{3}\right) p \in L^{1}[0,1]$ is nonnegative and $0 \leq \mu<1$, where $\mu=\int_{0}^{1} p(s) d s$.

\section{Denote}

$$
\begin{aligned}
& C^{+}[0,1]=\{u \in E: u(x) \geq 0, x \in[0,1]\} \\
& P=\{u \in E: u(x) \geq 0 \text { is concave and } u(x)=u(1-x), x \in[0,1]\} .
\end{aligned}
$$

It is easy to see that $P$ is a cone in $E$.

For any $y \in C^{+}[0,1]$, suppose that $u$ is a solution of the following BVP:

$$
\left\{\begin{array}{l}
u^{\prime \prime}(x)+f\left(x, y(x), y^{\prime}(x)\right)=0, \quad 0<x<1 \\
u(0)=u(1)=\int_{0}^{1} p(s) u(s) d s
\end{array}\right.
$$

Then we can easily get the solution:

$$
u(x)=\int_{0}^{1} H(x, t) f\left(t, y(t), y^{\prime}(t)\right) d t
$$

where

$$
H(x, t)=G(x, t)+\frac{1}{1-\mu} \int_{0}^{1} G(t, s) p(s) d s, \quad G(t, s)= \begin{cases}t(1-s), & 0 \leq t \leq s \leq 1 \\ s(1-t), & 0 \leq s \leq t \leq 1\end{cases}
$$


and

$$
\mu=\int_{0}^{1} p(s) d s
$$

During the process of getting the above solution, we can also know

$$
u^{\prime}(x)=\int_{0}^{1}(1-t) f\left(t, y(t), y^{\prime}(t)\right) d t-\int_{0}^{x} f\left(t, y(t), y^{\prime}(t)\right) d t
$$

for $x \in[0,1]$.

Lemma 2.1 If $\left(\mathrm{H}_{3}\right)$ is satisfied, the following results are true:

1. $H(x, t) \geq 0$, for $x, t \in[0,1] ; H(x, t)>0$ for $x, t \in(0,1)$.

2. $G(1-x, 1-t)=G(x, t), G(x, t) \leq G(x, x)$ for $x, t \in[0,1]$.

For any $y \in C^{+}[0,1], T: P \rightarrow E$ is defined

$$
(T y)(x)=\int_{0}^{1} H(x, t) f\left(t, y(t), y^{\prime}(t)\right) d t \quad \text { for } x \in[0,1] .
$$

Lemma 2.2 If $\left(\mathrm{H}_{3}\right)$ is satisfied, $T: P \rightarrow P$ is completely continuous, i.e., $T$ is continuous and compact. Moreover, $T$ is nondecreasing provided that $\left(\mathrm{H}_{2}\right)$ holds.

Proof For any $y \in P$, from the definition of $T y$, we know

$$
\left\{\begin{array}{l}
(T y)^{\prime \prime}(x)+f\left(x, y(x), y^{\prime}(x)\right)=0, \quad 0<x<1, \\
(T y)(0)=(T y)(1)=\int_{0}^{1} p(s)(T y)(s) d s .
\end{array}\right.
$$

Obviously, $T y$ is concave. From the expression of $T y$, combining with Lemma 2.1, we know that $T y$ is nonnegative on $[0,1]$. We now prove that $T y$ is symmetric about $\frac{1}{2}$.

For $x \in\left[0, \frac{1}{2}\right]$, then $(1-x) \in\left[\frac{1}{2}, 1\right]$, and

$$
\begin{aligned}
(T y)(1-x)= & \int_{0}^{1}\left(G(1-x, t)+\frac{1}{1-\mu} \int_{0}^{1} G(t, s) p(s) d s\right) f\left(t, y(t), y^{\prime}(t)\right) d t \\
= & \int_{0}^{1} G(1-x, t) f\left(t, y(t), y^{\prime}(t)\right) d t \\
& +\frac{1}{1-\mu} \int_{0}^{1} \int_{0}^{1} G(t, s) p(s) f\left(t, y(t), y^{\prime}(t)\right) d s d t \\
= & \int_{1}^{0} G(1-x, 1-t) f\left(1-t, y(1-t), y^{\prime}(1-t)\right) d(1-t) \\
& +\frac{1}{1-\mu} \int_{0}^{1} \int_{0}^{1} G(t, s) p(s) f\left(t, y(t), y^{\prime}(t)\right) d s d t \\
= & \int_{0}^{1} G(x, t) f\left(1-t, y(t),-y^{\prime}(t)\right) d t \\
& +\frac{1}{1-\mu} \int_{0}^{1} \int_{0}^{1} G(t, s) p(s) f\left(t, y(t), y^{\prime}(t)\right) d s d t
\end{aligned}
$$




$$
\begin{aligned}
& =\int_{0}^{1} G(x, t) f\left(t, y(t), y^{\prime}(t)\right) d t+\frac{1}{1-\mu} \int_{0}^{1} \int_{0}^{1} G(t, s) p(s) f\left(t, y(t), y^{\prime}(t)\right) d s d t \\
& =(T y)(x) .
\end{aligned}
$$

Similarly, we have

$$
(T y)(1-x)=(T y)(x) \quad \text { for } x \in\left[\frac{1}{2}, 1\right] .
$$

So, $T P \subset P$. The continuity of $T$ is obvious. We now prove that $T$ is compact. Let $\Omega \subset P$ be a bounded set. Then there exists $R$ such that

$$
\Omega=\{y \in P \mid\|y\| \leq R\} .
$$

For any $y \in \Omega$, we have

$$
0 \leq f\left(s, y(s), y^{\prime}(s)\right) \leq \max \left\{f\left(s, y, y^{\prime}\right) \mid s \in[0,1], y \in[0, R], y^{\prime} \in[-R, R]\right\}=: M
$$

Therefore, from (2.3), we have

$$
\|(T y)\|_{\infty} \leq M+\frac{\mu}{1-\mu} M=\frac{M}{1-\mu}, \quad\left\|(T y)^{\prime}\right\|_{\infty} \leq M .
$$

So, $\|(T y)\|$ is uniformly bounded. Now we prove $T y$ is equi-continuous. For $0 \leq x_{1} \leq$ $x_{2} \leq \frac{1}{2}$, we have

$$
\begin{aligned}
& \left|(T y)\left(x_{2}\right)-(T y)\left(x_{1}\right)\right| \\
& \quad=\left|\int_{0}^{1}\left(G\left(x_{2}, t\right)-G\left(x_{1}, t\right)\right) f\left(t, y(t), y^{\prime}(t)\right) d t\right| \\
& \quad \leq \begin{cases}\int_{0}^{1}\left|\left(x_{2}-x_{1}\right)(1-t)\right| f\left(t, y(t), y^{\prime}(t)\right) d t, & 0 \leq x_{1} \leq x_{2} \leq t \leq 1, \\
\int_{0}^{1}\left|t\left(x_{1}-x_{2}\right)\right| f\left(t, y(t), y^{\prime}(t)\right) d t, & 0 \leq t \leq x_{1} \leq x_{2} \leq 1, \\
\int_{0}^{1}\left|t\left(1-x_{2}\right)-x_{1}(1-t)\right| f\left(t, y(t), y^{\prime}(t)\right) d t, & 0 \leq x_{1} \leq t \leq x_{2} \leq 1 .\end{cases} \\
& \quad \leq M\left|x_{2}-x_{1}\right| .
\end{aligned}
$$

Moreover,

$$
\left|(T y)^{\prime}\left(x_{2}\right)-(T y)^{\prime}\left(x_{1}\right)\right|=\left|\int_{x_{1}}^{x_{2}} f\left(t, y(t), y^{\prime}(t)\right) d t\right| \leq M\left|x_{2}-x_{1}\right| .
$$

And the similar results can be obtained for $\frac{1}{2} \leq x_{1} \leq x_{2} \leq 1$ and $0 \leq x_{1} \leq \frac{1}{2} \leq x_{2} \leq 1$.

The Arzelà-Ascoli theorem guarantees that $T \Omega$ is relatively compact, which means $T$ is compact.

Finally, we show that Ty is nondecreasing about $y$.

For any $y_{i}(x) \in P(i=1,2)$ with $y_{1}(x) \leq y_{2}(x)$. By the properties of a cone, we have $y_{2}(x)-$ $y_{1}(x) \in P$ for $x \in[0,1]$. Then $y_{2}(x)-y_{1}(x) \geq 0$ is concave and symmetric about $\frac{1}{2}$. Therefore,

$$
\begin{cases}y_{2}^{\prime}(x) \geq y_{1}^{\prime}(x) & \text { for } x \in\left[0, \frac{1}{2}\right] \\ y_{2}^{\prime}(x) \leq y_{1}^{\prime}(x) & \text { for } x \in\left[\frac{1}{2}, 1\right]\end{cases}
$$


Hence, for $x \in\left[0, \frac{1}{2}\right]$, by $\left(\mathrm{H}_{2}\right)$ and the definition of $T y$, we have

$$
\begin{aligned}
\left(T y_{1}\right)(x)-\left(T y_{2}\right)(x)= & \int_{0}^{1} H(x, t) f\left(t, y_{1}(t), y_{1}^{\prime}(t)\right) d t \\
& -\int_{0}^{1} H(x, t) f\left(t, y_{2}(t), y_{2}^{\prime}(t)\right) d t \leq 0 .
\end{aligned}
$$

Furthermore, we have

$$
\begin{aligned}
\left(T y_{2}\right)^{\prime}(x)-\left(T y_{1}\right)^{\prime}(x)= & \int_{0}^{1}(1-s) f\left(s, y_{2}(s), y_{2}^{\prime}(s)\right) d s-\int_{0}^{1}(1-s) f\left(s, y_{1}(s), y_{1}^{\prime}(s)\right) d s \\
& +\int_{0}^{x} f\left(s, y_{1}(s), y_{1}^{\prime}(s)\right) d s-\int_{0}^{x} f\left(s, y_{2}(s), y_{2}^{\prime}(s)\right) d s .
\end{aligned}
$$

In order to prove $\left(T y_{2}\right)(x)-\left(T y_{1}\right)(x)$ is concave, we need to prove $\left(T y_{2}\right)^{\prime}(x)-\left(T y_{1}\right)^{\prime}(x)$ is nonincreasing. Let $0 \leq x_{1} \leq x_{2} \leq \frac{1}{2}$, then

$$
\begin{aligned}
& \left(T y_{2}\right)^{\prime}\left(x_{2}\right)-\left(T y_{1}\right)^{\prime}\left(x_{2}\right)-\left(T y_{2}\right)^{\prime}\left(x_{1}\right)+\left(T y_{1}\right)^{\prime}\left(x_{1}\right) \\
& =\int_{x_{1}}^{x_{2}} f\left(s, y_{1}(s), y_{1}^{\prime}(s)\right) d s-\int_{x_{1}}^{x_{2}} f\left(s, y_{2}(s), y_{2}^{\prime}(s)\right) d s \leq 0 .
\end{aligned}
$$

A similar result can be obtained for $x \in\left[\frac{1}{2}, 1\right]$. And it is easy to see that $\left(T y_{2}\right)(x)-\left(T y_{1}\right)(x)$ is symmetric about $\frac{1}{2}$. So, $\left(T y_{2}-T y_{1}\right) \in P$ and thus $T$ is nondecreasing.

\section{Existence and iterative of solutions for BVP (1.1) and (1.2)}

Theorem 3.1 Assume that $\left(\mathrm{H}_{1}\right)-\left(\mathrm{H}_{3}\right)$ hold. If there exist two positive numbers $a_{1}<$ a such that

$$
\sup _{x \in\left[0, \frac{1}{2}\right]} f(x, a, a) \leq a_{1}
$$

where $a$ and $a_{1}$ satisfy

$$
a \geq \max \left\{\frac{2 \mu}{9(1-\mu)}, \frac{1}{8}+\frac{\mu}{6(1-\mu)}, \frac{1}{2}\right\} a_{1},
$$

then problem (1.1) and (1.2) has a concave symmetric positive solution $w^{*}, v^{*} \in P$ with

$$
\begin{aligned}
& \left\|w^{*}\right\|_{\infty} \leq a \text { and } \quad \lim _{n \rightarrow \infty} T^{n} w_{0}=w^{*}, \quad \text { where } w_{0}(x)=a x(1-x)+\frac{3}{4} a, \\
& \left\|v^{*}\right\|_{\infty} \leq a \text { and } \quad \lim _{n \rightarrow \infty} T^{n} v_{0}=v^{*}, \quad \text { where } v_{0}(x)=0
\end{aligned}
$$

Proof We denote $P_{a}=\{w \in P:\|w\| \leq a\}$. In what follows, we first prove $T P_{a} \subset P_{a}$.

Let $w \in P_{a}$, then $0 \leq w(x) \leq \max _{x \in[0,1]} w(x)=\|w\|_{\infty} \leq a, \max _{x \in[0,1]}\left|w^{\prime}(x)\right|=w^{\prime}(0) \leq a$.

By assumption $\left(\mathrm{H}_{2}\right)$ and (3.1), for $x \in\left[0, \frac{1}{2}\right]$, we have

$$
0 \leq f\left(x, w(x), w^{\prime}(x)\right) \leq f(x, a, a) \leq \sup _{x \in\left[0, \frac{1}{2}\right]} f(x, a, a) \leq a_{1}
$$


For any $w(x) \in P_{a}$, by Lemma 2.2 , we know $T w \in P$ and, as a result,

$$
\begin{aligned}
\|T w\|_{\infty} & =(T w)\left(\frac{1}{2}\right) \\
& =\int_{0}^{1} G\left(\frac{1}{2}, t\right) f\left(t, w(t), w^{\prime}(t)\right) d t+\frac{1}{1-\mu} \int_{0}^{1} \int_{0}^{1} G(t, s) p(s) f\left(t, w(t), w^{\prime}(t)\right) d s d t \\
& \leq \frac{1}{8} a_{1}+\frac{a_{1}}{1-\mu} \int_{0}^{1} \int_{0}^{1} t(1-t) p(s) d s d t \\
& =\frac{1}{8} a_{1}+\frac{a_{1} \mu}{6(1-\mu)} \leq a
\end{aligned}
$$

and

$$
\left\|(T w)^{\prime}\right\|_{\infty}=(T w)^{\prime}(0)=\int_{0}^{1}(1-s) f\left(s, w(s), w^{\prime}(s)\right) d s \leq \frac{1}{2} a_{1} \leq a
$$

Hence, $\|T w\| \leq a$. Thus, we get $T P_{a} \subset P_{a}$. Let $w_{0}(x)=a x(1-x)+\frac{3}{4} a$ for $x \in[0,1]$, then $\left\|w_{0}\right\|=a$ and $w_{0}(x) \in P_{a}$. Let $w_{1}(x)=T w_{0}$, then $w_{1} \in P_{a}$. We denote

$$
w_{n+1}=T w_{n}=T^{n+1} w_{0} \quad(n=0,1,2, \ldots)
$$

Since $T P_{a} \subset P_{a}$, we have $w_{n} \in P_{a}(n=0,1,2, \ldots)$. From Lemma 2.2, $T$ is compact, we assert that $\left\{w_{n}\right\}_{n=1}^{\infty}$ has a convergent subsequence $\left\{w_{n_{k}}\right\}_{k=1}^{\infty}$ and there exists $w^{*} \in P_{a}$ such that $w_{n_{k}} \rightarrow w^{*}$. From the definition of $T,(3.1)$ and (3.2), we have

$$
\begin{aligned}
w_{1}(x) & =\left(T w_{0}\right)(x) \\
& =\int_{0}^{1}\left(G(x, t)+\frac{1}{1-\mu} \int_{0}^{1} G(t, s) p(s) d s\right) f\left(t, w_{0}(t), w_{0}^{\prime}(t)\right) d t \\
& =\int_{0}^{1} G(x, t) f\left(t, w_{0}(t), w_{0}^{\prime}(t)\right) d t+\frac{1}{1-\mu} \int_{0}^{1} \int_{0}^{1} G(t, s) p(s) f\left(t, w_{0}(t), w_{0}^{\prime}(t)\right) d s d t \\
& \leq \frac{1}{2} a_{1}\left(x-x^{2}\right)+\frac{\mu a_{1}}{6(1-\mu)} \\
& \leq a x(1-x)+\frac{3}{4} a=w_{0} .
\end{aligned}
$$

On the other hand, we notice that

$$
w_{0}^{\prime \prime}(x)-w_{1}^{\prime \prime}(x) \leq a_{1}-2 a \leq 0 .
$$

So, $w_{0}(x)-w_{1}(x) \in P_{a}$. By Lemma 2.2, we know $T w_{1} \leq T w_{0}$, which means $w_{2} \leq w_{1}, 0<$ $x<1$. By induction, $w_{n+1} \leq w_{n}, 0<x<1(n=0,1,2, \ldots)$. Hence, we assert that $w_{n} \rightarrow w^{*}$. Let $n \rightarrow \infty$ in (3.4) to obtain $T w^{*}=w^{*}$ since $\mathrm{T}$ is continuous. It is well known that the fixed point of the operator $T$ is the solution of BVP (1.1) and (1.2). Therefore, $w^{*}$ is a concave symmetric positive solution of BVP (1.1) and (1.2).

Let $v_{0}=0, x \in[0,1]$, then $v_{0} \in P_{a}$. Let $v_{1}=T v_{0}$, then $v_{1} \in P_{a}$, we denote

$$
v_{n+1}=T v_{n}=T^{n+1} v_{0}, \quad n=0,1,2, \ldots
$$


Similarly to $\left\{v_{n}\right\}_{n=1}^{\infty}$, we assert that $\left\{v_{n}\right\}_{n=1}^{\infty}$ has a convergent subsequence $\left\{v_{n_{k}}\right\}_{k=1}^{\infty}$ and there exists $v^{*} \in P_{a}$ such that $v_{n_{k}} \rightarrow v^{*}$. Now, since $v_{1} \geq v_{0}$, by Lemma 2.2, we know $T v_{1} \geq T v_{0}$, which means $v_{2} \geq v_{1}, 0<x<1$. By induction, $v_{n+1} \geq v_{n}, 0<x<1(n=0,1,2, \ldots)$. Hence, we assert that $v_{n} \rightarrow v^{*}, T v^{*}=v^{*}$, and $v^{*}(x)>0, x \in(0,1)$. Therefore, $v^{*}$ is a concave symmetric positive solution of BVP (1.1) and (1.2).

Remark The existence of a solution under the assumptions of Theorem 3.1 is just a consequence of Schauder's fixed point theorem. The monotone iterative technique adds the information about the approximation sequences.

Example Consider the following second-order boundary value problem with integral boundary conditions:

$$
\left\{\begin{array}{l}
u^{\prime \prime}(x)+\left[\frac{1}{80}+\frac{x^{\frac{1}{2}}(1-x)^{\frac{1}{2}}}{20}\right]\left[\left(u^{\prime}\right)^{2}+\ln (u(x)+1)+1\right]=0, \quad 0<x<1, \\
u(0)=u(1)=\int_{0}^{1} s u(s) d s .
\end{array}\right.
$$

And we have

$$
f(x, u, v)=\left[\frac{1}{80}+\frac{x^{\frac{1}{2}}(1-x)^{\frac{1}{2}}}{20}\right]\left[(v)^{2}+\ln (u+1)+1\right] .
$$

It is easy to check that the assumptions $\left(\mathrm{H}_{1}\right)-\left(\mathrm{H}_{3}\right)$ hold and $\mu=1 / 2$. Set $a=10, a_{1}=15$. Then we can verify that condition (3.1) is satisfied. Then applying Theorem 3.1, BVP (3.6) has a concave symmetric positive solution $w^{*}, v^{*} \in P$ with

$$
\begin{aligned}
& \left\|w^{*}\right\|_{\infty} \leq 10 \text { and } \lim _{n \rightarrow \infty} T^{n} w_{0}=w^{*}, \quad \text { where } w_{0}(x)=10 x(1-x)+\frac{15}{2} \text {, } \\
& \left\|v^{*}\right\|_{\infty} \leq 10 \text { and } \lim _{n \rightarrow \infty} T^{n} v_{0}=v^{*}, \quad \text { where } v_{0}(x)=0 \text {. }
\end{aligned}
$$

\section{Competing interests}

The authors declare that they have no competing interests.

Authors' contributions

The authors declare that the study was realized in collaboration with the same responsibility. All authors read and approved the final manuscript.

\section{Author details}

${ }^{1}$ College of Science, China Agricultural University, Beijing, 100083, P.R. China. ${ }^{2}$ The School of Statistics, Renmin University of China, Beijing, 100872, P.R. China.

\section{Acknowledgements}

The authors are highly grateful for the referees' careful reading and comments on this paper. The research is supported by Chinese Universities Scientific Fund (Project No. 2013QJ004).

Received: 15 February 2013 Accepted: 3 June 2013 Published: 25 June 2013

\section{References}

1. Gallardo, JM: Second order differential operators with integral boundary conditions and generation of semigroups. Rocky Mt. J. Math. 30, 1265-1292 (2000)

2. Karakostas, GL, Tsamatos, PC: Multiple positive solutions of some Fredholm integral equations arisen from nonlocal boundary-value problems. Electron. J. Differ. Equ. 30, 1-17 (2002)

3. Lomtatidze, A, Malaguti, L: On a nonlocal boundary-value problems for second order nonlinear singular differential equations. Georgian Math. J. 7, 133-154 (2000) 
4. $\mathrm{Ma}, \mathrm{H}$ : Symmetric positive solutions for nonlocal boundary value problems of fourth order. Nonlinear Anal. 68 , 645-651 (2008)

5. Boucherif, A: Second-order boundary value problems with integral boundary conditions. Nonlinear Anal. 70, 364-371 (2009)

6. Feng, M: Existence of symmetric positive solutions for a boundary value problem with integral boundary conditions. Appl. Math. Lett. 24, 1419-1427 (2011)

7. Wang, Y, Liu, G, Hu, Y: Existence and uniqueness of solutions for a second order differential equation with integral boundary conditions. Appl. Math. Comput. 216, 2718-2727 (2010)

8. Jankowski, T: Second-order boundary value problems with integral boundary conditions. J. Comput. Appl. Math. 147, 1-8 (2002)

9. Yao, Q: Existence and iteration of $\mathrm{n}$ symmetric positive solutions for a singular two-point boundary value problem. Comput. Math. Appl. 47, 1195-1200 (2004)

doi:10.1186/1687-2770-2013-150

Cite this article as: Pang and Tong: Symmetric positive solutions to a second-order boundary value problem with integral boundary conditions. Boundary Value Problems 2013 2013:150.

Submit your manuscript to a SpringerOpen ${ }^{\circ}$ journal and benefit from:

- Convenient online submission

- Rigorous peer review

- Immediate publication on acceptance

- Open access: articles freely available online

- High visibility within the field

- Retaining the copyright to your article

Submit your next manuscript at $>$ springeropen.com 